\title{
DSC+TG and XRD Study of Order-Disorder Transition in Nonstoichiometric Sr-Gd-Cobaltate
}

\author{
Sergei N. Vereshchagin ${ }^{* a}$, \\ Vyacheslav A. Dudnikov ${ }^{\mathrm{b}}$ and Leonid A. Solovyov ${ }^{\mathrm{a}}$ \\ ${ }^{a}$ Institute of Chemistry and Chemical Technology SB RAS \\ FRC "Krasnoyarsk Science Center SB RAS" \\ 50/24 Akademgorodok, Krasnoyarsk, 660036, Russia \\ ${ }^{b}$ Kirensky Institute of Physics \\ 50/38 Akademgorodok, Krasnoyarsk, 660036, Russia
}

Received 15.04.2016, received in revised form 27.05.2016, accepted 22.07.2016

\begin{abstract}
Process of interconversion of tetragonal $\mathrm{Sr}(0.8) \mathrm{Gd}(0.2) \mathrm{CoO}(3-d)$ (with ordered $\mathrm{Sr} / \mathrm{Gd}$ cations and anion vacancies) to cubic one (with disordered structure) was studied by X-ray structural and thermal analysis at 1100-1473 K. It was shown that the transformation is a first order diffuse phase transition. The ramp rate does not affect cation and anion vacancies disordering which is controlled by thermodynamic parameters of the processes in the solid whereas cubic to tetragonal transition is kinetically controlled. A theory of diffuse phase transition was applied to quantitatively analyze heat capacity temperature dependence.
\end{abstract}

Keywords: perovskite, order-disorder, phase transition, DSC.

DOI: 10.17516/1998-2836-2016-9-3-326-336.

(C) Siberian Federal University. All rights reserved

* Corresponding author E-mail address: snv@icct.ru 


\title{
Изучение фазового перехода порядок-беспорядок \\ в нестехиометрическом Sr-Gd-кобальтате \\ методами ДСК, ТГ и РСА
}

\author{
С.Н. Верещагин ${ }^{\mathrm{a}}$, \\ В.А. Дудников ${ }^{\tilde{}}$, Л.А. Соловьев ${ }^{a}$ \\ ${ }^{a}$ Институт химии и химической технологии СО РАН \\ ФИЦ «Красноярский научный иентр СО РАН» \\ Россия, 660036, Красноярск, Академгородок, 50/24 \\ ${ }^{6}$ Институт физики им. Л.В. Киренского СО РАН \\ Россия, 660036, Красноярск, Академгородок, 50/38
}

Методами термического и рентгеноструктурного анализа в интервале температур 11001473 К изучен процесс взаимопревращения тетрагонального перовскита $\operatorname{Sr}(0.8) \operatorname{Gd}(0.2)$ $\mathrm{CoO}(3-d)$ с упорядоченным расположением катионов $\mathrm{Sr} / \mathrm{Gd}$ и анионных вакансий в кубическую разупорядоченную модификаиию. Показано, что фазовое превращение протекает как размытый фазовый переход первого рода. Прочесс разупорядочения не зависит от скорости нагрева и контролируется термодинамическими характеристиками процессов в кристалле, тогда как процесс образования упорядоченной тетрагональной структуры контролируется кинетическими факторами. Проведен количественный анализ температурной зависимости теплоемкости на основе размытых фазовых переходов.

Ключевые слова: перовскит, порядок-беспорядок, фазовый переход, ДСК.

\section{Введение}

Замещенные редкоземельные кобальтаты $M_{x} L n_{1-x} \mathrm{CoO}_{3-\delta}(M-$ щелочно-земельный металл, $L n$ - лантаноид) со структурой перовскита - перспективные системы для применения в различных технологических процессах и устройствах [1], таких как топливные элементы [2], кислородпроводящие мембраны [3], газовые сенсоры [4]; они проявляют значительную активность в каталитических реакциях глубокого и парциального окисления углеводородов [5], активно исследуется их применение в процессах электрокатализа [6].

Такое разнообразие физико-химических свойств обусловлено особенностями перовскитной структуры $\mathrm{ABO}_{3}$, способной совмещать широкий спектр редкоземельных и щелочноземельных элементов, а также возможностью образования двойных перовскитов $\left(M_{z}^{\prime}{ }^{\prime}{ }^{\prime \prime}{ }_{1-z}\right)$ ( $\left(L n^{\prime} L n^{\prime \prime}\right)_{1-x} \mathrm{CoO}_{3-\delta}$. Известно, что при образовании двойных перовскитов $\mathrm{Sr}_{x} L n_{1-x} \mathrm{CoO}_{3-\delta}$ в зависимости от соотношения ионных радиусов катионов возможно различное равновесное распределение ионов $\mathrm{Sr}^{2+}$ и $\mathrm{Ln}^{3+}$. Для элементов $\mathrm{Ln}=\mathrm{La}-\mathrm{Nd}$ при всех температурах стабильна структура с полностью разупорядоченным распределением катионов $\mathrm{Sr}^{2+} / \mathrm{Ln}^{3+}$ по кристаллографическим позициям А структуры. Для элементов, радиус которых меньше, чем у $\mathrm{Nd}^{3+}$, разупорядоченные перовскиты существуют при высокой температуре, при низких темпера-

$$
-327-
$$


турах стабильной является структура с упорядоченным расположением катионов $\mathrm{Sr}^{2+}, \mathrm{Ln}^{3+}$ и анионных вакансий [7]. Однако для этих элементов разупорядоченные перовскиты $\mathrm{Sr}_{x} L n_{1-}$ ${ }_{x} \mathrm{CoO}_{3-\delta}$ могут быть получены в виде метастабильных фаз закаливанием высокотемпературных состояний [8].

Зафиксировано, что распределение катионов в А-позициях кристаллической структуры оказывает существенное влияние на свойства кобальтатов. Так, было продемонстрировано, что каталитическая активность монофазного соединения $\mathrm{Sr}_{0.8} \mathrm{Gd}_{0.2} \mathrm{CoO}_{3-\delta}$ в реакции глубокого окисления метана существенно выше при случайном распределении катионов $\mathrm{Sr}^{2+} / \mathrm{Gd}^{3+}$ по сравнению с образцом с упорядоченным расположением $\mathrm{Gd}^{3+}$ и $\mathrm{Sr}^{2+}$ в А-позиции $[8,9]$; упорядочение катионов приводило также к возникновению аномалии в магнитных свойствах [10]. Поэтому понимание деталей процесса фазового перехода порядок-беспорядок в двойных перовскитах и стабилизации метастабильных разупорядоченных состояний является необходимым элементом технологии получения систем с контролируемым уровнем упорядочения. Такие состояния зачастую обладают новыми свойствами, не проявляющимися у обычных перовскитов. В частности, содержащие свинец перовскиты - релаксоры - имеют чрезвычайно высокие значения диэлектрической константы за счет формирования наноупорядоченных областей в разупорядоченной матрице [11]. К настоящему времени накоплен огромный практический материал по физико-химическим свойствам упорядоченных/разупорядоченных перовскитов [12-15], однако практически отсутствуют исследования, непосредственно посвященные изучению процесса взаимопревращения соответствующих структур. Цель настоящей работы - изучение особенностей процесса упорядочения катионов $\mathrm{Sr}^{2+} / \mathrm{Gd}^{3+}$ на примере фазового перехода порядокбеспорядок в системе $\mathrm{Sr}_{0.8} \mathrm{Gd}_{0.2} \mathrm{CoO}_{3-\delta}$.

\section{Экспериментальная часть}

Поликристаллический образец $\mathrm{Sr}_{0.8} \mathrm{Gd}_{0.2} \mathrm{CoO}_{3-\delta}(\delta=0.37)$ был получен по стандартной керамической технологии. Стехиометрические количества $\mathrm{Gd}_{2} \mathrm{O}_{3}, \mathrm{Co}_{3} \mathrm{O}_{4}$ (чистота 99.9 \%) и $\mathrm{SrCO}_{3}$ (чистота 99 \%) тщательно смешивались в яшмовой ступке в этаноле. Полученная смесь отжигалась на воздухе при температуре $1473 \mathrm{~K}$ с тройным повторением циклов перетирание - прокалка. Спрессованные таблетки отжигались при той же температуре в течение 24 ч и охлаждались со скоростью 2 \%мин до комнатной температуры.

Рентгенофазовый и рентгеноструктурный анализы проводили с использованием дифрактометра PANalytical X'Pert PRO (CoK $\alpha$ ) в интервале углов $2 \theta 10-140^{\circ}$, съемка при высокой температуре осуществлялась в высокотемпературной камере Anton Paar HTK 1200N. Обработка результатов проведена с применением полнопрофильного анализа поликристаллических веществ по методу Ритвельда [16] и методу минимизации производной разности [17].

Синхронный термический анализ проводился на приборе Netzsch STA Jupiter 449C с масcспектральным анализатором Aeolos QMS 403C в потоке газовой смеси $20 \% \mathrm{O}_{2}$-Ar при атмосферном давлении в платиновых или алундовых тиглях с перфорированной крышкой. Исследовался монолитный поликристаллический образец размером около $1.5 \times 2 \times 1$ мм массой 16 мг. Качественный состав газов оценивали по изменению интенсивности ионов $\mathrm{c} \mathrm{m} / \mathrm{z}=18\left(\mathrm{H}_{2} \mathrm{O}\right)$, $28\left(\mathrm{~N}_{2}\right), 32\left(\mathrm{O}_{2}\right), 44\left(\mathrm{CO}_{2}\right)$. Величина теплового эффекта рассчитывалась из площади пика на кривой ДСК с применением коэффициентов чувствительности сенсора, определенных по ме-

$$
-328-
$$


тоду [18] из теплоемкости стандартного сапфирового диска. Дополнительно чувствительность проверена по теплоте плавления золота $\left(\mathrm{T}_{\mathrm{m}}=1335.2 \mathrm{~K}, \Delta \mathrm{H}=63.7\right.$ Дж/г), ошибка определения $\Delta \mathrm{H}$ составляла $4 \%$.

Содержание кислорода в исходном образце $\mathrm{Sr}_{0.8} \mathrm{Gd}_{0.2} \mathrm{CoO}_{2.63}$ установлено из убыли массы образца при его восстановлении водородом по методике [19], погрешность определения нестехиометрии $\delta$ составляла \pm 0.01 , изменение величины $\delta$ в циклах нагреваниеохлаждение рассчитывалось из потери массы (ТГ) относительно исходного содержания кислорода $\delta=0.37$.

\section{Результаты и обсуждение}

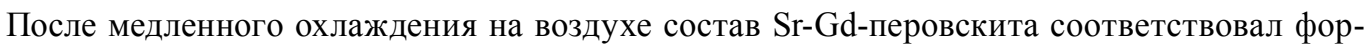
муле $\mathrm{Sr}_{0.8} \mathrm{Gd}_{0.2} \mathrm{CoO}_{2.63}$, а его структура, по данным рентгеноструктурного анализа (рис. $1 a$ ), соответствовала тетрагональной $I 4 / \mathrm{mmm}$ суперструктуре с упорядоченным расположением ионов $\mathrm{Sr}^{2+} / \mathrm{Gd}^{3+}$ и анионных вакансий (рис. 2б), аналогичной описанной в работе [7]. In-situ высокотемпературный РФА анализ показал, что выше 1373 К сверхструктурные рефлексы исчезали (рис. 1б) и формировался разупорядоченный нестехиометрический кубический перовскит, состав которого при $1473 \mathrm{~K}$ соответствовал формуле $\mathrm{Sr}_{0.8} \mathrm{Gd}_{0.2} \mathrm{CoO}_{2.51}$.

По данным термического анализа (рис. $3 a$ ), нагрев тетрагонального образца $\mathrm{Sr}_{0.8} \mathrm{Gd}_{0.2} \mathrm{CoO}_{2.63}$ в смеси $20 \% \mathrm{O}_{2}$-Ar протекал без видимых эффектов до $\mathrm{T}=773 \mathrm{~K}$, после чего начиналось монотонное снижение массы, обусловленное выделением кислорода. На фоне постоянного падения массы в температурном интервале $1343-1400 \mathrm{~K}$ наблюдался эндоэффект $\Delta \mathrm{H}=16.4 \pm 3.1$ Дж/г, который в соответствии с результатами высокотемпературного РФА может быть отнесен к фазовому переходу тетрагонального перовскита с упорядоченным расположением катионов $\mathrm{Sr}^{2+}$ $\mathrm{Gd}^{3+}$ в кубическую разупорядоченную модификацию. При последующем охлаждении происходило увеличение массы образца, а экзоэффект, соответствующий формированию упорядочен-

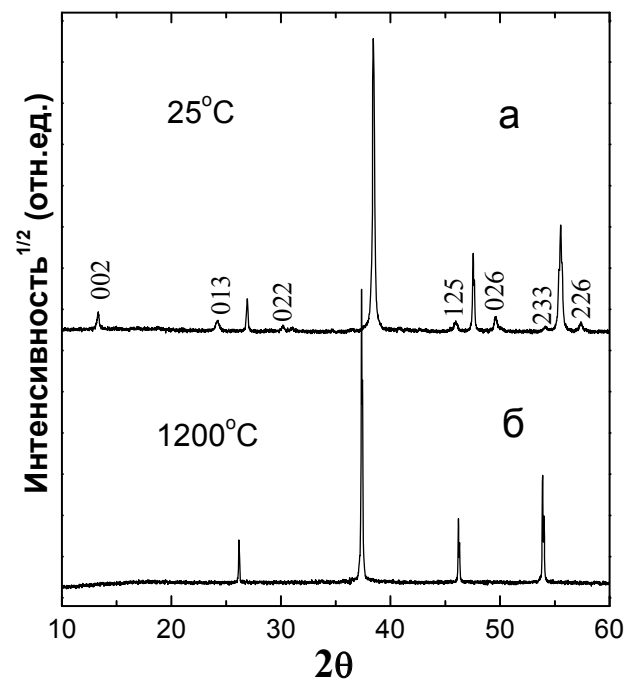

Рис. 1. Рентгенограммы образца $\mathrm{Sr}_{0.8} \mathrm{Gd}_{0.2} \mathrm{CoO}_{3-\delta}$ на воздухе: a $-25{ }^{\circ} \mathrm{C}, \delta=0.37 ; \sigma-1200^{\circ} \mathrm{C}, \delta=0.49$. Отмеченные рефлексы соответствуют сверхструктурным рефлексам тетрагонального перовскита 


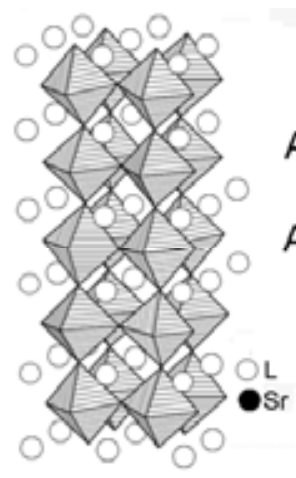

a

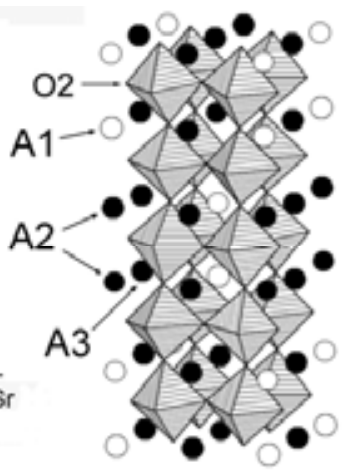

б

Рис. 2. Изображение кубической (а) и тетрагональной (б) структуры перовскита $\operatorname{Sr}_{0.8} \mathrm{Gd}_{0.2} \mathrm{CoO}_{3-\delta}$. Октаэдры соответствуют положению катионов $\mathrm{Co}^{\mathrm{n}+}$, белые и черные кружки обозначают положение катионов $\mathrm{Sr}^{2+} / \mathrm{Gd}^{3+}$. Ионы кислорода/анионные вакансии расположены в вершинах октаэдров, метки A1-A3 указывают места локализации катионов $\mathrm{Sr} / \mathrm{Gd}, \mathrm{O} 2$ - место преимущественной локализации кислородной вакансии в упорядоченной структуре
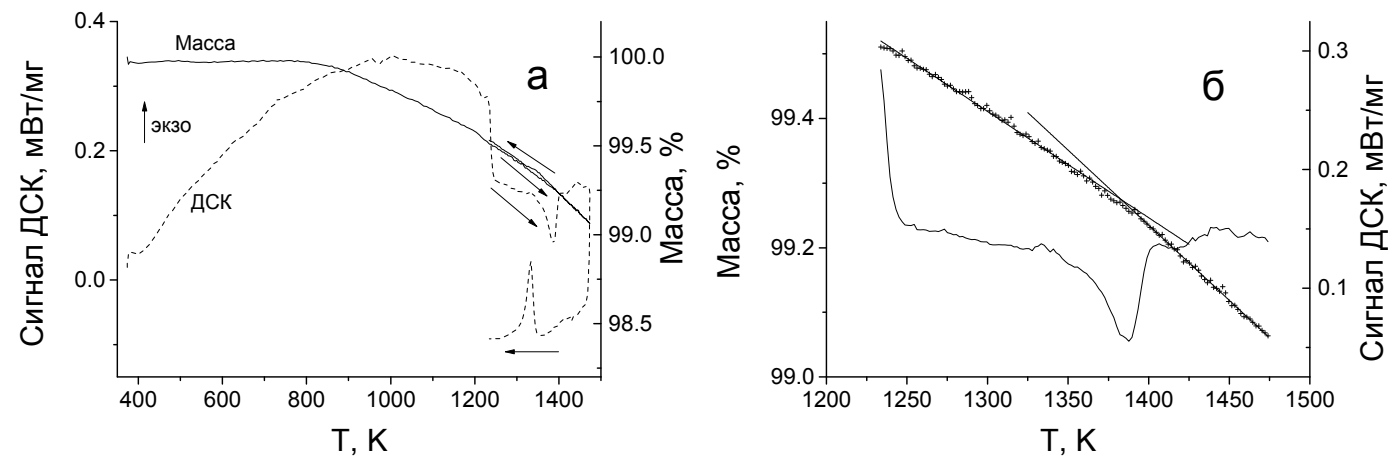

Рис. 3. а - ТГ (----) и ДСК(----) кривые для $\mathrm{Sr}_{0.8} \mathrm{Gd}_{0.2} \mathrm{CoO}_{2.63}$ в потоке смеси $20 \% \mathrm{O}_{2}$ - $\mathrm{Ar}$, алундовый тигель с перфорированной крышкой. Нагрев 20 \%мин до $1033 \mathrm{~K}$, далее нагрев до $1473 \mathrm{~K}$ и охлаждение до $1033 \mathrm{~K}$, $10 \%$ мин. Стрелки указывают направление изменения температуры; б - ДСК-сигнал (линия) и изменение массы (точки) вблизи температуры фазового превращения. Прямые линии показывают скорость изменения массы до и после перехода

ной структуры, наблюдался при 1354-1253 К. Важно отметить, что ход кривых ТГ практически совпадал при подъеме и снижении температуры (рис. $3 a$ ), что указывает на близкие состояния образца в циклах нагрев-охлаждение. Несмотря на то что при низких температурах разупорядоченный метастабильный перовскит содержит существенно больше кислорода по сравнению со стабильной упорядоченной модификацией $\left(\mathrm{Sr}_{0.8} \mathrm{Gd}_{0.2} \mathrm{CoO}_{2.71}\right.$ и $\mathrm{Sr}_{0.8} \mathrm{Gd}_{0.2} \mathrm{CoO}_{2.63}$ при 300-670 K соответственно, [8]), само превращение в присутствии $20 \% \mathrm{O}_{2}$ в газовой фазе протекало без заметного изменения массы. Различие наблюдалось только для скорости падения массы (выделения кислорода), которая увеличивалась при переходе упорядоченной фазы в разупорядоченную (рис. 3б), что согласуется с обнаруженным ранее фактом более высокой подвижности кислорода на образцах со случайным распределением катионов $\mathrm{Sr} / \mathrm{Gd}[8,9]$. 
Известно, что при использовании метода ДСК могут возникать методические погрешности, связанные с наличием термосопротивления в системе сенсор-тигель-образец, что выражается в искажении формы и смещении пиков ДСК при изменении скорости нагревания или охлаждения. Для учета этих эффектов была предложена методика [20], основанная на анализе зависимости $\mathrm{T}_{\max , \min }=f\left(\beta^{1 / 2}\right)$, где $\mathrm{T}_{\max , \min }-$ температуры максимума (минимума) пика ДСК; $\beta$ скорость изменения температуры. Экстраполяция этих зависимостей к величине $\beta=0$ позволяет определить методически не искаженные значения температур превращений. На рис. 4 приведены семейства кривых ДСК, полученные при различных скоростях сканирования $-2.5,5,10$ и 20 \%мин, а на вставке - соответствующая анаморфоза температур максимумов пиков. Из данных рисунка следует, что $\mathrm{T}_{\min }$ пика разупорядочения практически не менялась при изменении скорости сканировании $\beta$ (коэффициент корреляции Пирсона $\mathrm{r}=0.40$ незначим на уровне 0.05 , $\mathrm{N}=7$ ), тогда как для обратного перехода $\mathrm{T}_{\max }$ смещалась в область низких температур с ростом величины $\beta$. Независимость $\mathrm{T}_{\max }$ от $\beta$ для процесса разупорядочения указывает на отсутствие существенного вклада методической аппаратной составляющей в характеристики ДСК сигнала и позволяет утверждать, что наблюдаемые формы пиков обусловлены контролируемыми термодинамикой процессами в кристалле. В отличие от пика разупорядочения положение ДСК сигнала процесса перехода беспорядок-порядок зависело от скорости изменения температуры, что указывает на возможный вклад аппаратной составляющей или, что более вероятно, кинетических факторов (диффузии кислорода, скорости зародышеобразования) в процесс фазового превращения. Экстраполяция величин $\mathrm{T}_{\max , \min }$ к нулевой скорости сканирования $(\beta=0$, вставка рис. 4) показывает, что наблюдается существенный гистерезис в циклах нагреваниеохлаждение, что является одним из признаков, по которому наблюдаемый фазовый переход можно отнести к фазовым превращениям первого рода.

Дополнительная информация о процессе фазового перехода может быть получена из формы кривых температурной зависимости теплоемкости $\mathrm{C}_{\mathrm{p}}$ в области фазового превращения. Известно, что идеальные переходы первого рода имеют $\delta$-образную форму с разрывом зави-

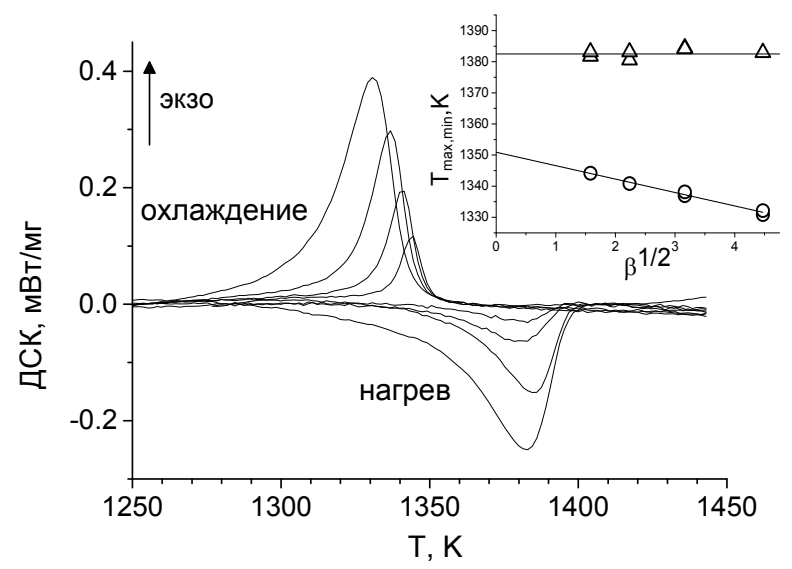

Рис. 4. ДСК кривые образца $\mathrm{Sr}_{0.8} \mathrm{Gd}_{0.2} \mathrm{CoO}_{2.63}$ (смесь $20 \% \mathrm{O}_{2}$ - $\mathrm{Ar}$, платиновый тигель с перфорированной крышкой) для скоростей сканирования $\beta=2.5(--), 5(---), 10(\cdots)$ и $20(-\cdot)$ \%мин. На вставке зависимость температуры максимума/минимума пика ДСК от $\sqrt{\beta} . \Delta-$ нагрев, О - охлаждение 
симости $\mathrm{C}_{\mathrm{p}}(\mathrm{T})$ в точке перехода. Однако для реальных кристаллов часто фазовые переходы протекают в некотором интервале температур. Для таких превращений, получивших название размытых фазовых переходов, вид $\mathrm{C}_{\mathrm{p}}(\mathrm{T})$ представляет собой симметричный колоколообразный пик $\Lambda$-типа. Для “чистых” фазовых переходов второго рода (ориентационных) характерна асимметричная $\lambda$-образная температурная зависимость $\mathrm{C}_{\mathrm{p}}(\mathrm{T})$ в окрестности фазового перехода; встречаются также промежуточные варианты между указанными случаями, приводящие к возникновению большей или меньшей асимметрии пиков теплоемкости. Возникновение размытого фазового перехода связывают с влиянием флуктуации состава кристалла или концентрации структурных дефектов, в результате чего критическая температура перехода также приобретает флуктурирующий характер [21]. Еще одно объяснение размывания перехода основано на предположении о взаимодействии структурных дефектов с межфазной границей, приводящее к коррелированному кооперативному характеру перемещения частиц в процессе перехода. Феноменологическая теория размытых фазовых переходов, развитая в работах [22, 23], позволяет количественно описать $\Lambda$-образные кривые на температурных зависимостях и определить основные параметры превращения. Исходя из термодинамического вида зависимости свободной энергии перехода в модели самосогласованного поля (1) было показано, что температурная зависимость аномальной теплоемкости $\Delta C_{p}(T)$, связанная с фазовым переходом первого рода, может быть представлена в виде зависимости (2):

$$
\Delta F(\varphi, T)=\left(U_{1} \varphi+U_{2}(1-\varphi)\right)+U_{0} \varphi(1-\varphi)+k T(\varphi \ln \varphi+(1-\varphi) \ln (1-\varphi))
$$

где $\varphi$ - доля частиц в фазовом состоянии $1 ; U_{1}, U_{2}$ - энергия частиц в фазовом состоянии 1 или 2; $U_{0} \varphi(1-\varphi)$ - энергия взаимодействия частиц.

$$
\Delta C_{p}(T)=\Delta C_{p, m} \frac{4 \exp \left(B\left(\frac{T}{T_{0}}-1\right)\right)}{\left(1+\exp \left(B\left(\frac{T}{T_{0}}-1\right)\right)\right)^{2}}
$$

где $T_{0}$ - критическая (характеристическая) температура перехода в отсутствие внешних полей и дефектов; $\Delta C_{p, m}$ - максимальное значение теплоемкости при $T=T_{0} ; B$ - атермический параметр.

При этом переход протекает в интервале температур $\Delta T$, а параметр $B$ связан с теплотой перехода $q_{0}$, плотностью кристалла $\rho$ и объемом элементарного превращения $\omega(3,4)$.

$$
\begin{aligned}
& \Delta T=\frac{4 T_{0}}{B}=\frac{4 k T_{0}^{2}}{\omega q_{0}}, \\
& B=\frac{\omega \rho q_{0}}{k T_{0}}, \\
& \Delta C_{p, m}=\frac{B q_{0}}{4 T_{0}} .
\end{aligned}
$$

В соответствии с этой теорией при фазовом переходе локализация флуктуаций в объеме происходит в виде зародышей новой фазы, объем которой увеличивается малыми порциями (элементарными объемами $\omega$ ), которые последовательно добавляются к новой фазе на межфазных границах. Величина $\omega$ является важной характеристикой механизма процесса форми- 
рования новой фазы. Так, в частности, для материалов, обладающих сегнетоэлектрическими свойствами, элементарные объемы превращения (области Кенцига) составляют $10^{4}-10^{5}$ нм [24], для фазового перехода первого рода в $\mathrm{Sm}_{1+\mathrm{x}} \mathrm{S}$ величина $\omega$ менялась в диапазоне 20-800 нм в зависимости от х [25].

Как было отмечено ранее в настоящей работе, вид кривой ДСК в области фазового превращения упорядоченного $\mathrm{Sr}_{0.8} \mathrm{Gd}_{0.2} \mathrm{CoO}_{3-\delta}$ в разупорядоченный перовскит не зависел от условий эксперимента (рис. 4), а определялся термодинамикой процессов в кристалле, что дает возможность применить описанный выше анализ формы пика $\Delta C_{p}$ для более полного понимания особенностей процесса разупорядочения. Для этого из кривой ДСК процесса разупорядочения (рис. $5 a$, точки $\times$ ) была выделена составляющая, соответствующая аномальной части теплоемкости $\Delta C_{p}$, связанная с фазовым переходом (рис. 56 , точки + ). Выделение проводилось путем преобразования измеряемого ДСК сигнала в шкалу теплоемкости $C_{p}=($ сигнал ДСК) $/ \beta$ и вычитанием базовой линии, соответствующей изменению теплоемкости системы до и после фазового превращения. Полученная температурная зависимость $\Delta C_{p}$ (рис. 5б) имела слегка асимметричную форму с затянутым низкотемпературным фронтом, что указывает на то, что исследуемый переход не является чисто фазовым превращением первого рода, а имеет более сложный характер. Возможной причиной этого служит предположение о промежуточной форме перехода [23], при котором энергия взаимодействия частиц в разных состояниях (уравнение 1 , второе слагаемое) сопоставима с энтропийным членом (уравнение 1 , третье слагаемое), что приводит к соответствующему искажению пика.

Однако детальное рассмотрение структуры $\mathrm{Sr}_{0.8} \mathrm{Gd}_{0.2} \mathrm{CoO}_{3-\delta}$ в различных состояниях позволяет выдвинуть альтернативное объяснение. Так, в разупорядоченной форме $\mathrm{Sr}_{0.8} \mathrm{Gd}_{0.2} \mathrm{CoO}_{3-\delta}$ катионы $\mathrm{Sr}^{2+} / \mathrm{Gd}^{3+}$ случайным образом распределены по А-позициям, а анионные (кислородные) вакансии и ионы $\mathrm{O}^{2-}$ - по кристаллографическим позициям кислорода (вершины октаэдров вокруг В-катиона, рис. $2 a$ ). В упорядоченном $\mathrm{Sr}_{0.8} \mathrm{Gd}_{0.2} \mathrm{CoO}_{2.63}$ существуют три различных A-позиции (A1-A3, рис. 26), которые заняты катионами $\mathrm{Sr}^{2+} / \mathrm{Gd}^{3+}$, причем положения $\mathrm{A} 2$ и $\mathrm{A} 3$ заняты исключительно ионами $\mathrm{Sr}^{2+}$, а в позиции $\mathrm{A} 1$ могут находиться как $\mathrm{Sr}^{2+}$, так и $\mathrm{Gd}^{3+}$. Кроме

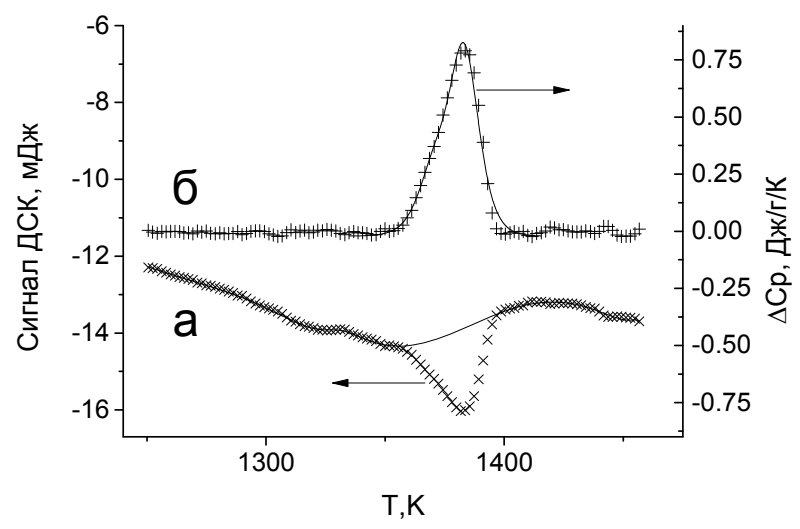

Рис. 5. а - фрагмент сигнала ДСК $(\times)$ и базовая линия для вычисления аномальной части теплоемкости вблизи области фазового перехода, нагрев 10\%мин, $20 \% \mathrm{O}_{2}$ - $\mathrm{Ar}$; б - температурная зависимость аномальной теплоемкости для кристалла $\mathrm{Sr}_{0.8} \mathrm{Gd}_{0.2} \mathrm{CoO}_{3-\delta}$ точки (+) - эксперимент, линия аппроксимация 
того, эта структура характеризуется также упорядоченным расположением кислородных вакансий, которые локализованы только в одном (положение О2, рис. 2б) из четырех кристаллографически различных позиций кислорода. Таким образом, переход упорядоченной структуры в разупорядоченную включает как минимум два, в общем случае независимых, процесса: (I) переход порядок-беспорядок в кислородной подрешетке, состоящий в рандомизации кислородных вакансий, и (II) перераспределение катионов гадолиния между тремя доступными позициями. Относительная независимость этих процессов иллюстрируется исследованиями системы $\mathrm{La}_{0.1} \mathrm{Sr}_{0.9} \mathrm{Co}_{0.9} \mathrm{Fe}_{0.1} \mathrm{O}_{3-\delta}[19]$, в которой продемонстрирован переход упорядоченной по типу браунмиллерита структуры в разупорядоченный перовскит без изменения катионной подрешетки. Кроме того, установлено, что значительный вклад в стабилизацию упорядоченного слоевого распределения А-катионов в $\mathrm{NaLaMgWO}_{6}$ вносит эффект Яна-Теллера, приводящий к смещению В-катионов [26]; перераспределения анионных вакансий хотя и протекают параллельно, но являются дополнительным, а не определяющим фактором. В этом случае наблюдаемая форма пика аномальной теплоемкости $\Delta C_{p}(T)$ будет суперпозицией двух смещенных по температуре превращений, связанных с неодновременностью процессов разупорядочения анионной и катионной подрешеток.

На рис. $5 б$ приведена теоретическая кривая, полученная аппроксимацией экспериментальных значений $\Delta C_{p}$ (точки + ) функцией $\Delta C_{p}=\Delta C_{p 1}+\Delta C_{p 2}$, где $\Delta C_{p 1}$ и $\Delta C_{p 2}-$ рассчитанные по уравнению (2) вклады анионной и катионной подрешеток. Вычисление параметров модели проведено методом нелинейной регрессии, оценки параметров и стандартные ошибки приведены ниже: $B_{l}=295 \pm 32, C_{p, m l}=0.27 \pm 0.11$ Дж/г/К, $T_{1}=1371 \pm 2 \mathrm{~K}$ и $B_{2}=338 \pm 50, C_{p, m 2}=0.78 \pm 0.12$ Дж/г/К, $T_{2}=1383 \pm 1$ К. Видно, что экспериментальные точки хорошо описываются предложенной моделью. Величина элементарного объема превращения, рассчитанная из уравнений (4) и (5), такова: $\omega_{1}=200$ нм$^{3}$ и $\omega_{2}=90$ нм$^{3}$, что соответствует 1.5-3 формульным единицам, учитывая стехиометрию кобальтата в точке перехода $\mathrm{Sr}_{0.8} \mathrm{Gd}_{0.2} \mathrm{CoO}_{2.54}$ и приняв плотность кристалла $\rho=5.53$ г/см ${ }^{3}$. При этом более высокая температура, скорее всего, соответствует процессу разупорядочения катионов, как менее подвижных частиц.

\section{Выводы}

Фазовый переход тетрагонального перовскита $\mathrm{Sr}_{0.8} \mathrm{Gd}_{0.2} \mathrm{CoO}_{3-\delta}$ с упорядоченным расположением катионов $\mathrm{Sr}^{2+} / \mathrm{Gd}^{3+}$ в кубическую разупорядоченную модификацию, происходящий в интервале температур 1331-1383 K (20\% О글 нагрев/охлаждение 2-20 \%мин), протекает как размытый фазовый переход первого рода. Процесс разупорядочения катионов и кислородных вакансий протекает легко и не зависит от скорости нагрева, состояние системы в процессе разупорядочения контролируется термодинамическими характеристиками процессов в кристалле, а не кинетикой образования зародышей новой фазы и их роста. В рамках модели размытых фазовых переходов по теории самосогласованного поля температурная зависимость аномальной части теплоемкости в области фазового превращения хорошо описывается в предположении о независимости процессов разупорядочения катионной и анионной подрешеток.

В отличие от перехода порядок-беспорядок обратный процесс образования упорядоченной тетрагональной структуры контролируется кинетическими факторами (скоростями зарож- 
дения зародышей и/или их роста). Таким образом, получение кобальтатов с различной степенью упорядоченности необходимо осуществлять из полностью разупорядоченного перовскита ( $\mathrm{T}>1398$ К для изученного состава) путем варьирования температурных программ охлаждения/ отжига и состава газовых сред.

\section{Благодарности}

Исследование выполнено при финансовой поддержке Российского фонда фундаментальных исследований, Правительства Красноярского края, Красноярского краевого фонда поддержки научной и научно-технической деятельности в рамках научного проекта 16-43-240505 «p_a».

\section{Список литературы}

1. Tao S.W. and Irvine J. T. S. Metal Oxides: Chemistry and Applications, ed. J. L. G. Fierro. Boca Raton: CRC Press, 2006. P. 739.

2. Inagaki T., Miura K., Yoshida H., Maric R., Ohara S., Zhang X., Mukai K., Fukui T. Highperformance electrodes for reduced temperature solid oxide fuel cells with doped lanthanum gallate electrolyte: II. $\mathrm{La}(\mathrm{Sr}) \mathrm{CoO}_{3}$ cathode. J. Power Sources. 2000. Vol. 86, P. 347-351.

3. Chen C.H., Bouwmeester H.J.M., Doorn R.H.E., Kruidhof H., Burggraaf A.J. Oxygen permeation of $\mathrm{La}_{0.3} \mathrm{Sr}_{0.7} \mathrm{CoO}_{3-\delta}$. Solid State Ionics. 1997. Vol. 98 , P. 7-13.

4. Michel C.R., Martínez A.H., Huerta-Villalpando F., Morán-Lázaro J.P. Carbon dioxide gas sensing behavior of nanostructured $\mathrm{GdCoO}_{3}$ prepared by a solution-polymerization method. Journal of Alloys and Compounds. 2009. Vol. 484, P. 605-611.

5. Pena M. A. and Fierro J.L.G. Chemical Structures and Performance of Perovskite Oxides. Chem. Rev. 2001. Vol. 101, P. 1981-2018.

6. Jun L., Ling Z. and Guanzhong L. Activation of Methane over Perovskite Catalysts. Ind. Eng. Chem. Res. 2009. Vol.48, P. 641-646.

7. James M., Cassidy D., Goossens D.J. and Withers R.L. The phase diagram and tetragonal superstructures of the rare earth cobaltate phases $L_{1-x} \mathrm{Sr}_{x} \mathrm{CoO}_{3-\delta}\left(L n=\mathrm{La}^{3+}, \mathrm{Pr}^{3+}, \mathrm{Nd}^{3+}, \mathrm{Sm}^{3+}, \mathrm{Gd}^{3+}, \mathrm{Y}^{3+}\right.$, $\mathrm{Ho}^{3+}, \mathrm{Dy}^{3+}, \mathrm{Er}^{3+}, \mathrm{Tm}^{3+}$ and $\mathrm{Yb}^{3+}$ ). J. Solid State Chem. 2004. Vol. 177, P. 1886-1895.

8. Vereshchagin S.N., Solovyov L.A., Rabchevskii E.V., Dudnikov V.A., Ovchinnikov S.G. and Anshits A.G. Methane oxidation over A-site ordered and disordered $\mathrm{Sr}_{0.8} \mathrm{Gd}_{0.2} \mathrm{CoO}_{3-\delta}$ perovskites. Chemical Communications. 2014. Vol. 50, P. 6112-6115.

9. Vereshchagin S. N., Solov'ev L. A., Rabchevskii E. V., Dudnikov V. A., Ovchinnikov S. G., Anshits A. G. New method for regulating the activity of $\mathrm{ABO}_{3}$ perovskite catalysts. Kinetics and Catalysis. 2015. Vol. 56, P. 640-645.

10. Dudnikov, V. A.; Orlov, Yu. S.; Gavrilkin, S. Yu.; et al. Effect of Gd and Sr Ordering in A Sites of Doped $\mathrm{Gd}_{0.2} \mathrm{Sr}_{0.8} \mathrm{CoO}_{3 \text {-delta }}$ Perovskite on Its Structural, Magnetic, and Thermodynamic Properties. $J$. Phys. Chem. C. 2016. Vol. 120, P. 13443-13449.

11. Bhalla A.S., Guo R., Roy R. The perovskite structure - a review of its role in ceramic science and technology. Mat. Res. Innovat. 2000. Vol. 4, P. 3-26.

12. Liu R., Xuan Y., Jia Y.Q.. Ordering and disordering in (A'A') (B'B')O3-type perovskite compounds. Materials Chemistry and Physics. 1998. Vol. 57, P. 81-85. 
13. Mogni L., Prado F., Jiménez C., Caneiro A. Oxygen order-disorder phase transition in layered $\mathrm{GdBaCo}_{2} \mathrm{O}_{5+\delta}$ perovskite: Thermodynamic and transport properties. Solid State Ionics. 2013. Vol. 240, P. $19-28$.

14. Yin Q., Lin Y.S.. Beneficial effect of order-disorder phase transition on oxygen sorption properties of perovskite-type oxides. Solid State Ionics. 2007. Vol. 178, P. 83-89.

15. Yang Z.H., Lin Y.S. Synergetic thermal effects for oxygen sorption and order-disorder transition on perovskite-type oxides. Solid State Ionics. 2005. Vol. 176 , P. 89-96.

16. Reitveld H. A profile refinement method for nuclear and magnetic structures. Journal of Applied Crystallography. 1969. Vol. 2, P. 65-71.

17. Solovyov L.A. Full-profile refinement by derivative difference minimization. Journal of Applied Crystallography. 2004. Vol. 37, P. 743-749.

18. Thermal analysis; differential thermal analysis; principles. DIN 51007:1994-06.

19. Conder K., Pomjakushina E., Soldatov A., and Mitberg E. Oxygen content determination in perovskite-type cobaltates. Materials research bulletin. 2005. Vol. 40. P. 257-263 .

20. Illers K.-H. Die ermittlung des schmelzpunktes von kristallinen polymeren mittels wärmeflusskalorimetrie (DSC). European Polymer Journal. 1974. Vol. 10, P. 911-916.

21. Moreira R. L., Lobo R. P. S. M. Phenomenological Study of Diffuse Phase Transitions. Journal of the Physical Society of Japan. 1992. Vol. 61, PP. 1992-1995.

22. Малыгин Г.А. Размытые мартенситные переходы и пластичность кристаллов с эффектом памяти формы. Успехи физических наук. 2001. Т. 171, C. 187-212. [Malygin G.A. Diffuse martensite transitions and the plasticity of crystals with the shape memory effect. Uspekhi fizicheskikh nauk. 2001. Vol. 171. P. 187-212 (In Russ.)]

23. Malygin G.A. Analysis of the parameters of a smeared orientational transition at 250-260 K in C-60 crystals. Physics of the solid state. 2001. Vol. 43, P. 1989-1994.

24. Кенциг В. Сегнетоэлектрики и антисегнетоэлектрики. М., изд-во иностр. лит., 1960. 234 c. [Kanzig W. Ferroelectrics and antiferroelectrics (Solid state physics, vol. 4) . New York, 1957. $117 \mathrm{p}]$.

25. Egorov V. M., Kuzuya T., Kaminskii V. V., Hirai Sh., Sharenkova N. V. Specific Features of the Structure of Semiconducting SmS Polycrystals in the Homogeneity Region. Physics of the solid state. 2012. Vol. 54. P. 48-52.

26. Knapp M. C., Woodward P. M. A-site cation ordering in AA'BB'O6 perovskites. Journal of Solid State Chemistry. 2006. Vol. 179. P. 1076-1085. 